\title{
Silane depletion dependent ion bombardment and material quality of microcrystalline silicon deposited by VHF-PECVD
}

\author{
A. Feltrin, G. Bugnon, F. Meillaud, J. Bailat, and C. Ballif \\ Institute of Microtechnology (IMT), University of Neuchatel, Rue A.-L. Breguet 2, 2000 Neuchatel, Switzerland.
}

\section{Introduction}

Microcrystalline silicon is a composite material embedding silicon nanocrystals in an amorphous matrix [1]. It is a material that has attracted much research efforts in the photovoltaic domain [2], because of its potential for integration in a tandem cell concept as bottom cell with an amorphous silicon top cell. Efficiencies of micromorph tandem cells and modules well above $10 \%$ have thus been demonstrated [3]. However, due to its complex structure that depends on deposition conditions $[1,4]$ and substrate properties [5], and due to the difficulty of characterizing plasma deposition regimes, the impact of these parameters on the microcrystalline material quality is still an open field of research.

In this paper, microcrystalline silicon thin films are deposited in different conditions of silane depletion following a recent publication [6] and the material quality is investigated. It is shown that by simply reducing the hydrogen flow, the microcrystalline material quality can be greatly improved. This improvement is attributed to reduced ion bombardment energy in high depletion regimes, leading to lower defect densities in the microcrystalline intrinsic layer.

\section{Experimental}

Microcrystalline silicon layers were deposited by very high frequency (VHF) plasma enhanced chemical vapor deposition (PECVD) at $40.68 \mathrm{MHz}$ in an industrial KAI-S type reactor based on the plasmabox concept [7], using silane and hydrogen as gas precursors. During deposition, the peak to peak voltage $V_{p p}$ was measured on the RF driven electrode using a calibrated high impedance capacitive gauge connected to an oscilloscope. The samples were analyzed by micro Raman spectroscopy [8]. Solar cells were deposited in the $p-i-n$ configuration in the same reactor in a single chamber process on $\mathrm{ZnO}$ coated glass substrates [9]. The same doped layers and a $100 \mathrm{~nm}$ thin intrinsic buffer layer at the $\mathrm{p} / \mathrm{i}$ interface were used in all depositions and only the 1.2 $\mu \mathrm{m}$ thick bulk intrinsic layers were varied. The solar cell surface was $0.25 \mathrm{~cm}^{2}$ and the solar cells were characterized by I-V measurements using a WACOM sun simulator and by spectral response measurements to determine the external quantum efficiency.

\section{Results and Discussion}

Microcrystalline silicon intrinsic layers were deposited in different silane depletion regimes at 1.2 mbar and 2.0 mbar. These relatively low pressures were chosen in order to minimize polysilane and powder formation during deposition [10]. At 1.2 mbar, the input silane flow was fixed at $50 \mathrm{sccm}$ and two different depletion regimes were studied, varying the hydrogen flow between $1200 \mathrm{sccm}$ and $200 \mathrm{sccm}$, thus varying initial silane concentrations between $4 \%$ and $20 \%$. At $2.0 \mathrm{mbar}$, silane and hydrogen flows were both $75 \mathrm{sccm}$ ( $50 \%$ silane concentration). As has been shown previously [6,11], microcrystalline material can be deposited at high initial silane concentrations $c$, provided that the depletion $D$ in the plasma discharge is sufficiently large so that the ratio between the silane radical and atomic hydrogen $c_{p}$ remains small [12]:

$$
c_{p}=c \cdot(1-D)
$$

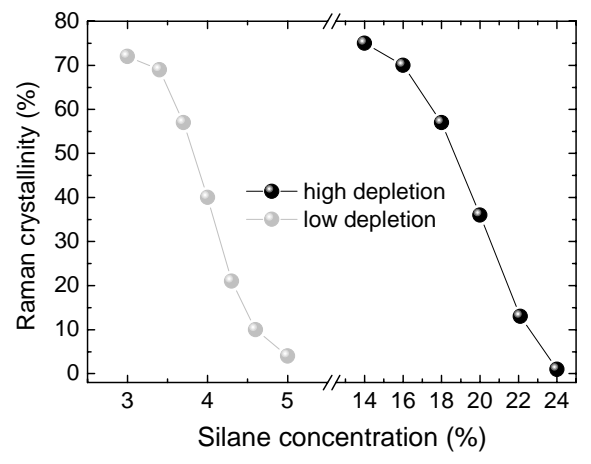

Fig. 1: Raman crystallinity as a function of input silane concentration in the two silane depletion regimes at 1.2 mbar. RF input power density is 0.15

$\mathrm{W} / \mathrm{cm}^{2}$. The transition under high depletion conditions is much larger in agreement with Eq. (1).

High depletion can be achieved either by increasing the RF input power or by increasing the residence time of the gas molecules in the reactor (i.e. decreasing the hydrogen flow).

Thus, in the following of this paper, the deposition regime around $4 \%$ initial silane concentration will be labeled low depletion regime, whereas the deposition regimes at higher initial silane concentrations will be called high depletion regime. In Figure 1, the 
transition at 1.2 mbar between amorphous and highly microcrystalline material is displayed as a function of silane concentration $c$ (i.e. at constant depletion $D$ ). Both curves were obtained with a low RF input power density of $0.15 \mathrm{~W} / \mathrm{cm}^{2}$. As can be noted from the two different scales on the horizontal axes, the width of the transition is much larger in the high depletion regime. This can be understood using equation (1) and assuming that $c_{p}$ is directly related to the crystallinity of the film [12]: for increasingly large values of the depletion $D$, a larger range of initial silane concentration $c$ has to be spanned in order to cover the same range in $c_{p}$, and therefore in crystallinity. The same equation can be used to show that the width of the transition from amorphous to highly microcrystalline material as a function of RF input power density (i.e. at constant input silane concentration $c$ ) will be different in the two cases as well. According to that equation, a higher silane concentration $c$ will yield a narrower transition region as a function of RF power density. Figure 2 displays the result in the two deposition regimes.

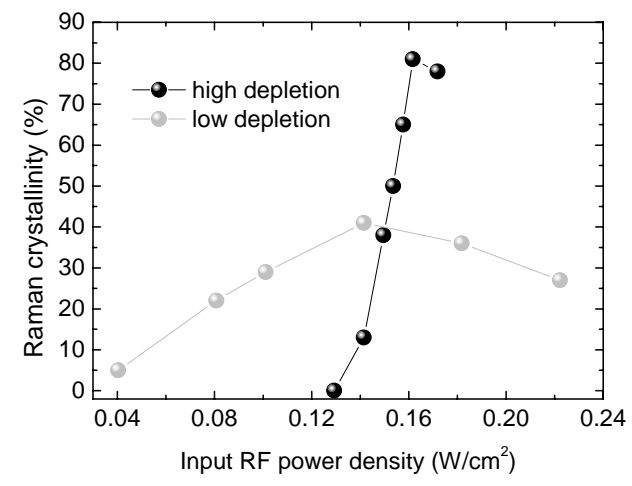

Fig. 2: Raman crystallinity as a function of input RF power density in the two depletion regimes at 1.2 mbar. The transition under high depletion conditions is very narrow in agreement with Eq. (1).

Two facts can be observed: first the transition region in the high depletion regime is clearly much smaller than in the low depletion regime; second, in the low depletion regime the crystallinity fraction increases slowly and even decreases for power densities higher than $0.14 \mathrm{~W} / \mathrm{cm}^{2}$. A decrease of crystallinity cannot be explained within the frame of equation (1) as higher RF power, increasing the depletion, should favor the growth of material with a higher crystalline fraction. It has been proposed in the past that ion bombardment is an important factor determining the microcrystalline material quality $[1,13,14]$ and that it affects the crystallinity as well [1]. Indeed, if the same data in Figure 2 are displayed as a function of the plasma potential $V_{p} \approx V_{p p} / 4$ which is proportional to ion energy accelerated through the plasma sheath and bombarding the substrate [15], it can be noted that in the high depletion regime the plasma voltage is significantly lower than in the low depletion regime (see Figure 3). Taking $V_{p}=100 \mathrm{~V}$ and typical values for collision cross sections at 1.2 mbar [15], the ion energy can be roughly estimated to $\sim 10 \mathrm{eV}$, a value very close to the threshold where ion damage induces film amorphization [1].

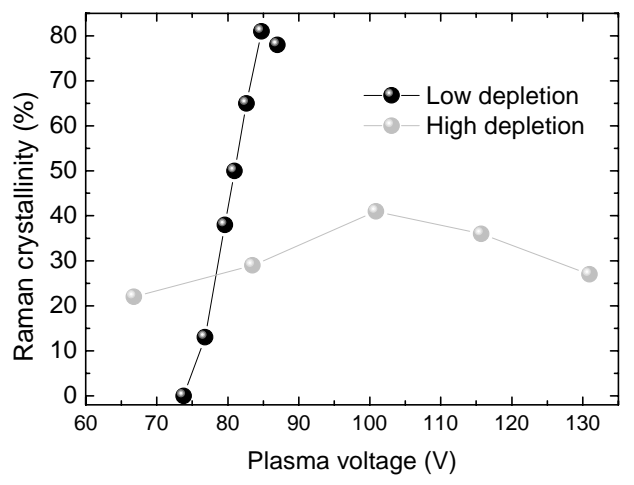

Fig. 3: Raman crystallinity as a function of plasma voltage in the two silane depletion regimes at 1.2 mbar.

This drop in the plasma voltage is not completely clear, but it can safely be attributed to a change of the plasma impedance associated to the higher silane concentration since the values of the external RF matching network are not the same in the two deposition regimes. It is possible that due to the increased silane concentration and gas residence time, some of the dissociated silane molecules form polysilane chains and that these macromolecules charge negatively. This would lower the electron density and the plasma potential, although simultaneous changes in the electron temperature cannot be excluded.

In support of the fact that in the high depletion regime the lower plasma voltage indeed corresponds to lower ion bombardment energy, $1.2 \mu \mathrm{m}$ thick intrinsic layers with comparable microcrystalline fraction around $60 \%$ have been deposited in the two regimes and integrated in p-i-n solar cell structures. The purpose here was not to optimize the solar cells in the two deposition regimes, but to use the solar cells as a diagnostic device in order to evaluate the material quality of the intrinsic layers. The I-V characteristics are displayed in Figure 4. It appears very clearly that the solar cell deposited in the high depletion regime has much better values of open circuit voltage, fill factor and short circuit current than the one deposited in the low depletion regime indicating an improved material quality. The solar 
cell efficiency increases from $3.4 \%$ to $6.6 \%$. This improvement is attributed to the lower ion bombardment energy, which has been shown to affect the microcrystalline material quality $[1,13,14]$. This result has been obtained despite the higher deposition rate $(6.5 \AA /$ s compared to $2.9 \AA / \mathrm{s})$ which typically tends to worsen the material quality [12].

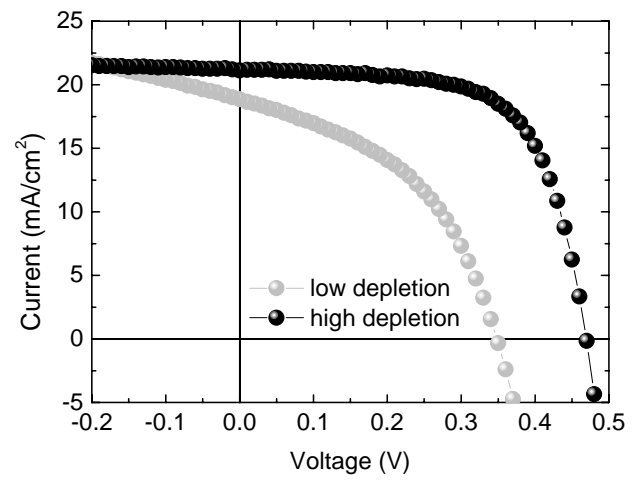

Fig. 4: IV characteristics of the solar cells deposited in the two silane depletion regimes at 1.2 mbar.

Figure 5 shows that the loss in short circuit current comes mainly from the red and infrared portion of the spectrum, whereas at shorter wavelengths the spectral response is almost identical. This is mainly due to the use of a common thin intrinsic buffer zone after the $p$ layer whereas the difference in the red and infrared portion of the spectrum reinforces the previous observations that in the low depletion regime the material quality is inferior.

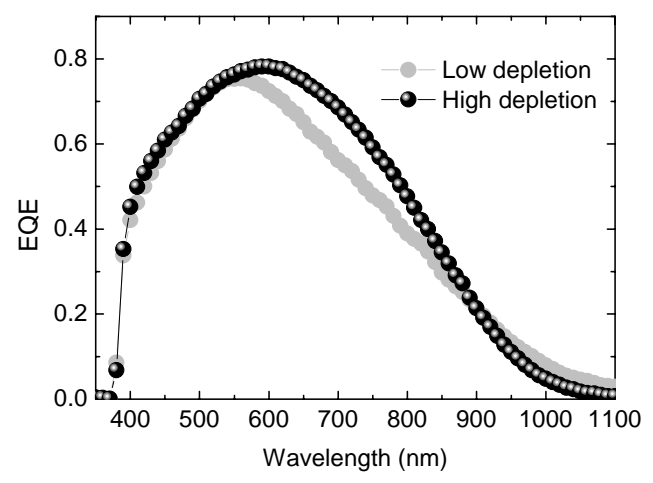

Fig. 5: Spectral response of the solar cells deposited in the two silane depletion regimes at 1.2 mbar.

Upon increasing the deposition pressure to $2.0 \mathrm{mbar}$ and the silane concentration to $50 \%$, it was possible to further increase the deposition rate to $9.0 \AA / \mathrm{s}$, a value interesting for applications in industry. The solar cell parameters (not shown) are very similar to the cell deposited in high depletion conditions at 1.2 mbar and the efficiency is $6.4 \%$ in these conditions.
However at these pressures powder formation becomes a relevant phenomenon. It limits further gains in deposition rate and might affect the quality of the deposited layer as well. Future investigations will be based on the findings described in this paper and on modifications to the reactor to further increase the growth rate as well as powder formation threshold and thus improve the material quality and cell efficiency.

\section{Conclusions}

In conclusion, a systematic study of microcrystalline thin films in the transition region has been performed under different conditions of silane depletion, input RF power and pressure. It has been shown that under high depletion conditions the material quality and the cell performance improve. This is attributed to the reduction of ion bombardment energy as the silane depletion is increased.

\section{Acknowledgements}

The authors acknowledge useful discussions with B. Strahm, A. Howling and C. Hollenstein.

\section{References}

[1] M. Kondo, Sol. Energy Mat. \& Sol. Cells 78, 543 (2003).

[2] J. Meier, R. Fluckiger, H. Keppner et al., Appl. Phys. Lett. 65, 860 (1994).

[3] M. A. Green, K. Emery, Y. Isikawa et al., Prog. Photovolt. 15, 425 (2007).

[4] U. Kroll, J. Meier, A. Shah et al., J. Appl. Phys. 80, 4971 (1996).

[5] E. Vallat-Sauvain, J. Bailat, J. Meier et al., Thin Solid Films 485, 77 (2005).

[6] B. Strahm, A.A. Howling, L. Sansonnens et al., Sol. Energy Mat. \& Sol. Cells 91, 495 (2007).

[7] A. Shah, J. Meier, A. Buechel et al., Thin Solid Films 502, 292 (2006).

[8] E. Vallat-Sauvain, C. Droz, F. Meillaud et al., J. Non-Cryst. Solids 352, 1200 (2006).

[9] S. Fay, L. Feitknecht, R. Schluchter et al., Sol. Energy Mat. \& Sol. Cells 90, 2960 (2006).

[10] Ch. Hollenstein, Plasma Phys. Control. Fusion 42, R93 (2000).

[11] M.N. Van den Donker, B. Rech, F. Finger, et al., Prog. Photovolt. 15, 291 (2007).

[12] B. Strahm, A.A. Howling, L. Sansonnens et al., Plasma Sources Sci. Technol. 16, 80 (2007).

[13] A. Gordijn, L Hodakova, J.K. Rath et al., J. Non-Cryst. Solids 352, 1868 (2006).

[14] T Matsui, A. Matsuda, M. Kondo, Sol. Energy Mat. \& Sol. Cells 90, 3199 (2006).

[15] M. Liebermann, A. Lichtenberg, Principles of Plasma Discharge and Material Processing, Wiley, New York, 1994. 